\title{
Preparation and characteristic of triolein-embedded composite sorbents for water purification
}

\author{
Jinxian Huo, Huijuan Liu, Jiuhui Qu*, Zijian Wang, Jiantuan Ge, Haining Liu \\ State Key Laboratory for Environmental Aquatic Chemistry, Research Center for Eco-Environmental Sciences, \\ Chinese Academy of Sciences Beijing 100085, PR China
}

Received 5 September 2004; received in revised form 2 December 2004; accepted 7 December 2004

\begin{abstract}
The novel triolein-embedded composite sorbents for removing persistent organic pollutants (POPs) in water were developed. Comprehensive structural characteristics of the composite sorbents were characterized using scanning electron microscope (SEM), fourier transform infrared spectrum (FT-IR) and X-ray photoelectron spectrum (XPS). It is suggested that triolein/cellulose acetate (CA) viscous syrup can be deposited on the surface of silica gel granules, because the silica gel granules have the large enough aperture to make the polymer penetrate into. Triolein is also embedded into a hybrid membrane perfectly. Dieldrin is regarded as a representative compound of POPs. Sorption experiments in solutions with constant concentrations show that adsorption capacity increased with increased triolein content in composite adorbents. Dieldrin removal could reach $80 \%$ at $50 \mathrm{ng} / \mathrm{l}$ initial concentration, which is near the environmental level.
\end{abstract}

(C) 2004 Elsevier B.V. All rights reserved.

Keywords: Triolein; Cellulose acetate (CA); Composite sorbent; Persistent organic pollutants (POPs); Dieldrin

\section{Introduction}

Persistent organic pollutants (POPs) as a set of organic compounds possess toxic characteristics. They are also persistent, liable to bioaccumulation and prone to long-range atmospheric transport. Moreover, they can result in adverse environmental and human health effects in locations near and far from their sources [1]. POPs released to the environment can travel through air and water to regions far from their original sources. In these distant regions, POPs can concentrate in living organisms, including humans, to levels that have the potential to injure the human health and/or the environment. The most common contaminated media of POPs include sediment, air and water [2]. Due to their lower concentration in water, from nanogram per litre to picogram per litre [3,4], POPs are especially difficult to be removed from water.

The most prevalent techniques in water treatment, such as photocatalysis [5], ozone-oxidation [6], biological degra-

\footnotetext{
* Corresponding author. Tel.: +86 10 62849151; fax: +86 1062923558 .

E-mail address: jhqu@mail.rcees.ac.cn (J. Qu).
}

dation [7] do a poor job in removing POPs from aqueous solutions because POPs resist photocatalysed, chemical and biological degradation. Sorption is a dominant and effective technique in removing organic pollutants from aqueous solutions, but its continued viability is greatly dependent on the development of novel sorptive materials [8]. Currently, active carbon adsorption is extensively used to remove organic pollutants from water [9]. Due to nonselectivity of active carbon to all organic pollutants and its tendency to desorption after saturation, however, it is difficult to remove trace or ultratrace POPs near the environmental level by sorption of active carbon. Activated carbon cannot adsorb organic halogen whose concentration is below $5 \mu \mathrm{g} / \mathrm{l}$ [10].

Current research is limited to analysis and identification, ecological effect, environmental monitoring, impact on human health and reproduction [11-16]. There are very little researches on the removal of trace POPs from water, especially on the developing of purificatory materials for POPs in water. Recent developed composite adsorbents have nothing to do with POPs, such as cationic MCM-41 for aromatic compounds [17], macroporous silica-based polymeric ma- 
terial for strontium [18], polyacrylic acid-bound iron oxide magnetic nanoparticles for methylene blue [19]. Therefore, the development of novel materials is urgent in order to improve water quality.

The object of this study is aimed at developing a novel composite sorbent for the removal of trace POPs in water. Triolein has a high accumulating capacity $\left(10^{5}-10^{7}\right)$ for trace POPs in water [20,21]. Cellulose acetate (CA) is a particularly useful polymer for preparing hybrid materials because it can be easily molded into different forms, such as membranes, fibers and spheres [22]. Furthermore, its hydrophilicity improves accessibility of aqueous solutions to the surface of the film. Mesoporous silica gel granules were selected as a supporter because mesoporous materials possess pores large enough to readily allow the penetration of polymer chains [23]. Furthermore, silica gel is prone to be chemically and physically modified. The novel composite sorbents were prepared with these pollution-free and environmental-friendly raw materials.

In this study, dieldrin was taken as a representative POPs because of its common characteristics of POPs, such as low solubility in water, nonpolarity and lipophilia. Sorption experiments were carried out in order to test adsorption capacity of three composite adsorbents. Sorption experiments at nanogram level were set to test the removal capacity for dieldrin dissolved in water at concentrations similar to those found in actual water bodies.

\section{Experimental section}

\subsection{Materials}

The FNG-II type water-tolerant silica gel with 2-4 mm diameter was obtained from Special Silica Gel Factory (Qingdao, China). Cellulose acetate ( 2.5 degree of acetylation) was purchased from Chemical Reagent Corporation (Shanghai, China). Triolein (purity, 99\%) was purchased from Sigma (St. Louis, MO, USA). Materials for the preparation of the composite sorbents also included 1,4-dioxane (A.R.) and acetone (A.R.). Dieldrin (purity, 98.6\%) was purchased from Accustandard Inc. (125 Market Street, New Haven, CT 06513).

\subsection{Instrumentations}

Scanning electron microscope (SEM) images were acquired using a HITACHI S-450 scanning electron microscope. The cross-section of the sample was prepared by freezing-fracturing in liquid nitrogen. A GIKOIB-3 sputter coater with a gold-palladium target was used for coating gold on the surface of samples.

The IR spectrum was obtained using a Spectrum GX FT-IR system from Perkin-Elmer. The attenuated total reflectance technique was used in the sample analysis. The wave number was between $4000 \mathrm{~cm}^{-1}$ and $650 \mathrm{~cm}^{-1}$ and scanning times were 16.
Specific surface area, pore volume and pore diameter were obtained by an ASAP 2000 surface analyzer (Micromeritics Co., USA) and liquid $\mathrm{N}_{2}$ was used as sorbate.

The X-ray photoelectron spectra (XPS) were recorded by the Japan Rigaku Dmax/gA diffractometer using an Xray source $(\mathrm{Al} \mathrm{K \alpha}, 1486.6 \mathrm{eV})$ with a 45.0 source analyzer angle. Samples for XPS analysis were prepared by freezing-desiccation for 3 days.

HITACHI F-3000 (Japan) was used to measure the triolein content in water that leaked out from composite adsorbents.

\subsection{Preparation of the composite sorbents}

The composite sorbents were prepared according to the following steps:

(1) First, the oxygen within acetone was removed by using nitrogen. Next $17 \mathrm{~g}$ of CA, different triolein contents (triolein versus CA) and other additives, such as 1,4dioxane, were added together in $80 \mathrm{ml}$ of acetone. The mixture was kept at $35^{\circ} \mathrm{C}$ for 2 days and stirred in order to admix uniformly. Finally, viscous syrups were sonicated for $30 \mathrm{~min}$ in order to enhance triolein dispersion [24]. Thus, viscous syrups with different triolein loadings were well prepared and homogeneous phases were obtained. Miscibility and solid-state structure for blends of triolein and CA were observed through SEM images (Figs. 1 and 2). Hybrid plat membranes were prepared as analytic samples. ATR-FTIR spectra (Fig. 3) were also obtained to identify the miscibility of triolein and CA.

(2) Silica gel granules were modified in order to enhance cementation between silica gel granules and the hybrid membranes. In a conical flask, $2 \mathrm{~g}$ CA was dissolved into $100 \mathrm{ml}$ acetone. Silica gel was added to the $\mathrm{CA} /$ acetone solution. After excessive solution was spilled out, acetone in the silica gel evaporated, and the modified silica gel desiccated in a freezing and vacuum manner for 3 days. In order to find out the optimum modified conditions, different dipping time and sonication time were considered. Subsequently, aperture analysis by BET and element analysis by X-ray photoelectron spectrum (XPS) were carried out. XPS results are shown in Table 1.

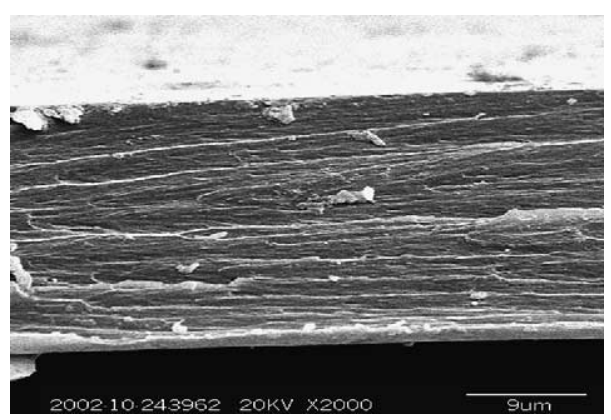

Fig. 1. SEM image of a pure CA membrane cross-section. 


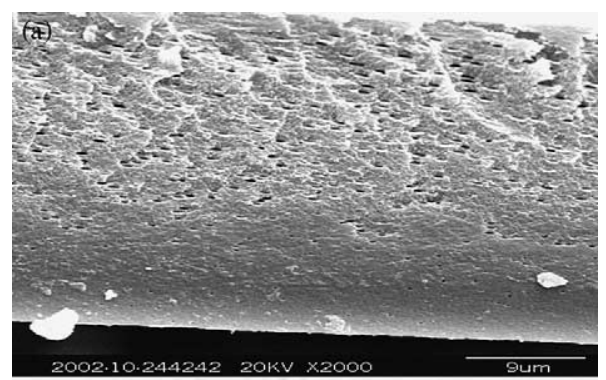

(b)

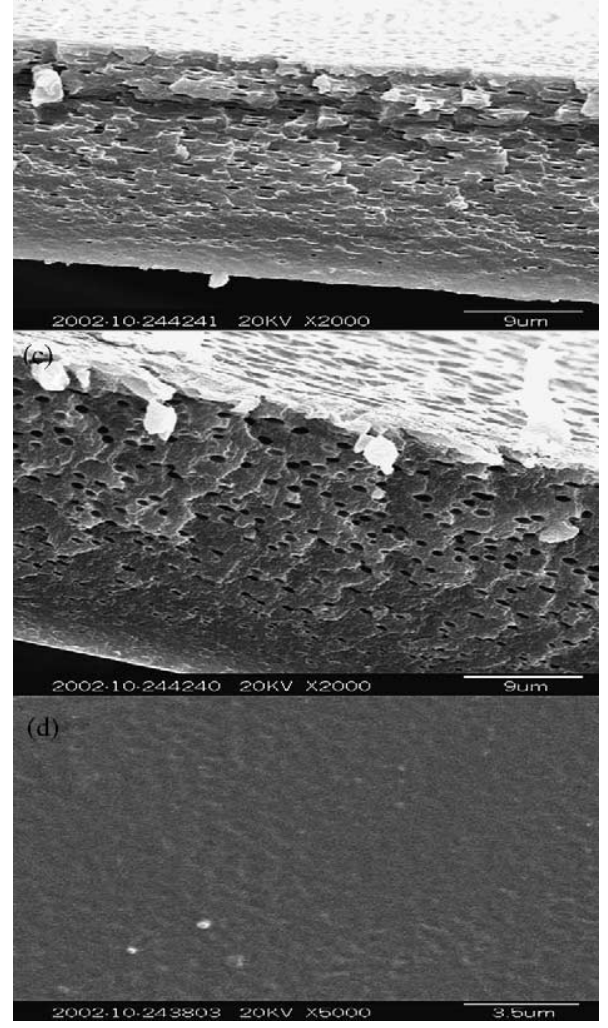

Fig. 2. SEM images of hybrid membranes with different triolein loadings: (a) triolein $/ \mathrm{CA}=0.06$, cross-section; (b) triolein $/ \mathrm{CA}=0.11$, cross-section; (c) triolein $/ \mathrm{CA}=0.17$, cross-section; (d) triolein $/ \mathrm{CA}=0.17$, top surface.

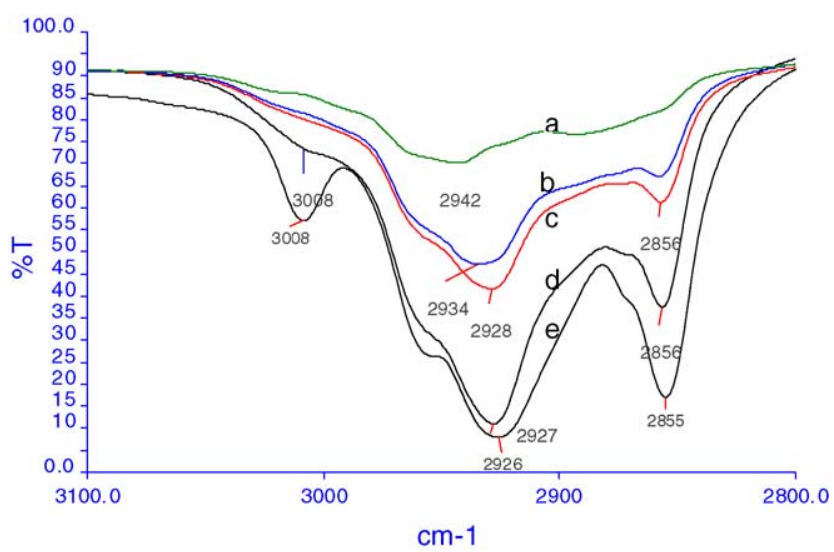

Fig. 3. ATR-FTIR spectra of the composite membrane with different triolein loadings: (a) pure $\mathrm{CA}$; (b) triolein $/ \mathrm{CA}=0.06$; (c) triolein $/ \mathrm{CA}=0.11$; (d) triolein $/ \mathrm{CA}=0.17$; (e) pure triolein.
Table 1

Surface element analysis by XPS

\begin{tabular}{lrlrl}
\hline & $\mathrm{C}_{1 \mathrm{~s}}$ & $\mathrm{O}_{1 \mathrm{~s}}$ & $\mathrm{Si}_{2 \mathrm{p}}$ & Others \\
\hline Silica gel & 7.6 & 65.4 & 27.0 & 0 \\
Dipping for 15 min & 57.4 & 37.9 & 4.7 & 0 \\
Dipping for 30 min & 58.9 & 38.0 & 3.1 & 0 \\
Dipping for 120 min & 53.8 & 37.3 & 8.9 & 0 \\
Ultrasonicating for 15 min & 56.2 & 40.1 & 3.7 & 0 \\
Ultrasonicating for 30 min & 58.4 & 35.7 & 0.5 & 5.4 \\
\hline
\end{tabular}

(3) The modified silica gel was immersed into viscous syrup for $30 \mathrm{~min}$, and then degassed by ultrasonic irradiation in order to remove any trapped air bubbles [23]. Redundant solution was removed and acetone evaporated. Subsequently, the granular silica gel coated with viscous syrup desiccated, while, integrated spherical granules were obtained. Finally, composite sorbents were washed with distilled water to remove dissoluble impurities. The TOC of the leachate was determined using a Phoenix 8000 Total Organic Carbon Analyzer (Tekmar-Dohrmann Co., USA). After exchanging the water up to 10 times over 3day period, the TOC of the leachate was near that of distilled water. With these procedures, three composite sorbents (STA000, STA014, STA021) were successfully prepared. Composite sorbents were desiccated in a freezing and vacuum manner for 3 days before the elements of the composite sorbent surface were analyzed by XPS. There were different triolein loadings with 0 of triolein/CA ratio to STA000, 0.14 to STA014 and 0.21 to STA021. $\mathrm{C}_{1 \mathrm{~s}}$ and $\mathrm{Si}_{2 \mathrm{p}}$ spectra of STA021 obtained by XPS are shown in Fig. 5a and b, respectively. Leakage of bound chemicals in composite adsorbents was measured by photoluminescence method.

\subsection{Uptake experiments}

Sorption experiments were carried out in triplicate with three kinds of composite sorbents in batch mode. Fresh solution was prepared with distilled water free of detectable dieldrin throughout the course of the uptake experiments. Ten grams of composite sorbent (wet weight) was added at the start of the run to a $500 \mathrm{ml}$ conical flask containing $400 \mathrm{ml}$ solution. Dieldrin concentration in aqueous solutions was $200 \mu \mathrm{g} / \mathrm{l}$ and $50 \mathrm{ng} / \mathrm{l}$, respectively. The solution was agitated for a predetermined time using a horizontal shaker operated at $160 \mathrm{rmp}$ at $25^{\circ} \mathrm{C}$.

Ten millilitres measured samples at different time intervals were withdrawn from the aqueous solution and transferred into $25 \mathrm{ml}$ extraction funnel with $0.5 \mathrm{~g}$ of anhydrous sodium chloride, an anti-emulsification agent. The samples were extracted with $1 \mathrm{ml} n$-hexane for three times. The samples were shaking for $10 \mathrm{~min}$. After delaminating, liquid extract was passed through an anhydrous sodium sulfate column and transferred into a $5 \mathrm{ml}$ volumetric flask. All liquid extracts were integrated and fixed at a constant volume of $5 \mathrm{ml}$. A HP5890 capillary gas chromatography-electron cap- 
ture detector (GC-ECD) system was used for analysis. Linear range was controlled between $5 \mu \mathrm{g} / \mathrm{l}$ and $50 \mu \mathrm{g} / \mathrm{l}$.

One microlitre aliquot of sample that was injected into a splitless injector $\left(250^{\circ} \mathrm{C}\right)$ was analyzed under the following conditions: A HP-5 fused silica capillary column $(30 \mathrm{~m} \times 0.32 \mathrm{~mm}$, i.d., film thickness of $0.17 \mu \mathrm{m})$ was fitted in the instrument. The ECD was maintained at $280^{\circ} \mathrm{C}$. The $\mathrm{GC}$ oven was temperature programmed as follows: $85^{\circ} \mathrm{C}$, isothermal for $2 \mathrm{~min}$, then the temperature was raised at $10^{\circ} \mathrm{C} / \mathrm{min}$ to $180^{\circ} \mathrm{C}$ and held at $180^{\circ} \mathrm{C}$ for $15 \mathrm{~min}$, followed by $4{ }^{\circ} \mathrm{C} / \mathrm{min}$ to $280^{\circ} \mathrm{C}$ and held at $280^{\circ} \mathrm{C}$ for $10 \mathrm{~min}$. Highpurified nitrogen was used as the carrier gas at a head pressure of $16 \mathrm{psi}$.

\section{Results and discussion}

\subsection{Immiscibility and structure of triolein/CA blends}

$\mathrm{CA}$ as a rigid thermoplastic $\left(T_{\mathrm{g}}=78^{\circ} \mathrm{C}\right)$ is soluble in acetone and can form a smooth and defect-free film upon evaporation by phase transition. Fig. 1 showed the SEM image of the cross-section of a pure CA flat membrane. SEM image of the topside (no shown) revealed a relatively smooth surface, free from obvious cracks, holes, or other defects.

Topside (air side) images of hybrid membranes with different triolein loadings were similar to that of pure CA membrane. Fig. $2 \mathrm{~d}$ was the topside image of the plat membrane with 0.17 triolein/CA ratio. It was obvious that no triolein exposed on topside of the hybrid membranes. It could be explained that aggregated rate of CA into air was faster than that of triolein. Cross-section images of hybrid membranes (Fig. 2a-c) with different triolein loadings revealed smaller droplets of triolein when triolein/CA ratio were 0.06 and 0.11 . Whereas, when triolein/CA ratio was 0.17 , triolein droplets became larger. Droplets were hardly observed within $10 \mu \mathrm{m}$ thick of layer from top surface when triolein/CA ratio was 0.06 . Increased triolein contents in hybrid membranes leaded in thinning of this layer. From the analysis of the SEM images, it was evident that triolein could be embedded into CA within an appropriate triolein loading. In the mean time, phase separation took place, so CA is immiscible with triolein.

Subsequently, ATR-FTIR was used to analyze miscibility of triolein and CA. ATR-FTIR spectra also identified that CA was immiscible with triolein. All these spectra from Fig. 3 were parts of whole spectra between $4000 \mathrm{~cm}^{-1}$ and $650 \mathrm{~cm}^{-1}$. Absorption peak in pure CA was not presented. Strong absorption peaks in pure triolein at $3002 \mathrm{~cm}^{-1}, 2926 \mathrm{~cm}^{-1}, 2855 \mathrm{~cm}^{-1}$ were presented. The peak at $3002 \mathrm{~cm}^{-1}$ was the stretching vibration absorption peak of $=\mathrm{C}-\mathrm{H}$ and the peaks at $2926 \mathrm{~cm}^{-1}, 2855 \mathrm{~cm}^{-1}$ were those of $\mathrm{C}-\mathrm{H}$, which presented the absorption peaks of double bond and long-chain alkanes of triolein. Other three curves were the FTIR spectra of hybrid membranes containing different triolein loadings. The peaks at $2926 \mathrm{~cm}^{-1}$ and $2855 \mathrm{~cm}^{-1}$ were stronger with increasing triolein loading, but the position of the peaks did not change. $=\mathrm{C}-\mathrm{H}$ peak at $3002 \mathrm{~cm}^{-1}$ presented obviously. All these results identified that triolein physically mixed with CA without any chemical bonding. It further showed that accumulated capability of triolein did not changed when triolein was embedded into CA.

\subsection{Modification of silica gel}

By aperture analysis, silica gel granule possessed pores $(112 \AA)$ large enough to readily allow the penetration of polymer chains, resulting in better wetting and dispersion. After being modified with $\mathrm{CA} /$ acetone solution, it could be obtained that average pore size of silica gel did not change, pore volume decreased from $0.71 \mathrm{~cm}^{3} / \mathrm{g}$ to $0.65 \mathrm{~cm}^{3} / \mathrm{g}$ and specific surface area from $250.69 \mathrm{~cm}^{2} / \mathrm{g}$ to $230.12 \mathrm{~cm}^{2} / \mathrm{g}$, which indicated that CA had anchored into granules. From surface element analysis data of Table 1,30 min were needed to complete the modification. Ultrasonic irradiation could enhance anchor. Too long time was unnecessary because adhesive agent within silica gel granules would dissolve due to the effect of acetone, accordingly silica will be exposed to the granular surface.

\subsection{Characterization of composite sorbents}

Characterizations of composite sorbent surface were evaluated by BET method and XPS. Fig. 4 was the curve of sorption and desorption on STA021 and silica gel. STA000 and STA014 presented same curves (no shown) as that of STA021. Fig. 4 suggests that sorption and desorption curve of STA021 was greatly different from that of silica gel after silica gel was coated with triolein/CA membrane. Sorption and desorption of silica gel answers for BET sorption model well. By analyzing BET sorption and desorption of STA021, highly purified nitrogen began to be adsorbed when relative pressure reached to 0.8 , whereas desorption hardly took place. So composite adsorbents coated with membrane did not agree with

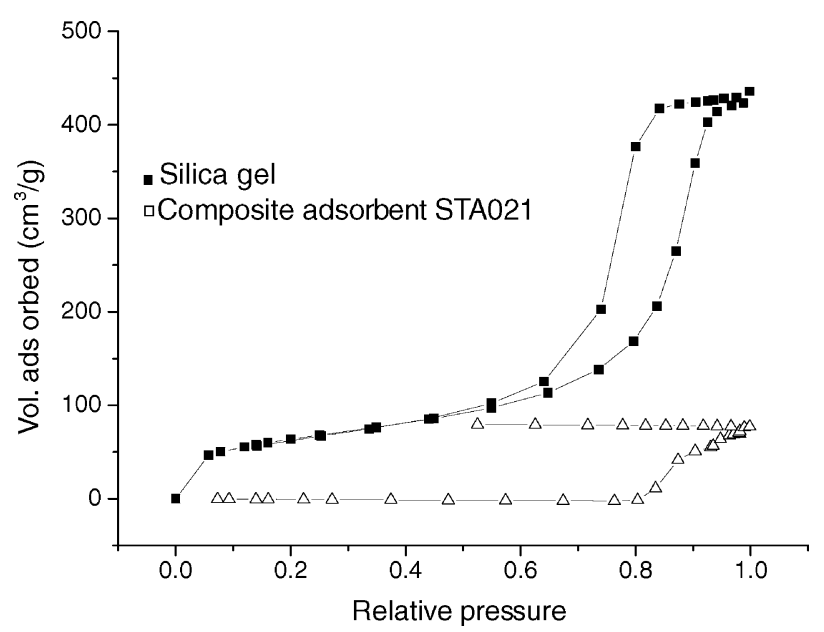

Fig. 4. Nitrogen sorption and desorption isotherm of STA021 and silica gel. 
BET sorption model. This could be explained by structural analysis of composite adsorbents. The inner of composite sorbent was the silica gel granule with $112 \AA$ pore size, and the hybrid membrane coated to it presented asymmetrical structure with a compact CA outer surface layer. It was only needed to conquer the resistance of the compact CA surface layer when sorption of $\mathrm{N}_{2}$ was carried on. However, when desorption was carried on, conquered resistance integrated the resistance of silica gel supporter and loose and compact layer of hybrid membrane, which leaded to the increase of resistance. In addition, due to the liquid-droplet state of triolein in membrane, pores in compact layer were obstructed when triolein intended to tranfer to the surface in condition of outer pressure of granule smaller than inner pressure of that. Irreversible sorption consequently took place to $\mathrm{N}_{2}$. XPS measurements were also performed to identify the surface compositions and characterizations of the three sorbents. Our deduction was based on $\mathrm{Si}_{2 \mathrm{p}}$ spectra would not present if dense membrane was absolutely coated onto the surface of silica gel granules and graphite spectra would also not present if triolein was not exposed to the surface of composite sorbents. Fig. 5a and $\mathrm{b}$ were XPS spectra for $\mathrm{C}_{1 \mathrm{~s}}$ and $\mathrm{Si}_{2 \mathrm{p}}$ of composite sorbents STA021. The spectra of another two sorbents were not shown because they had same spectra as STA021. $\mathrm{C}_{1 \mathrm{~s}}$ spectra showed that half peak width of composite adsorbent was large. They were $\mathrm{C}-\mathrm{O}$ at $285.7 \mathrm{eV}, \mathrm{C}-\mathrm{OH}$ at $286.74 \mathrm{eV}$, $\mathrm{C}-\mathrm{O}-\mathrm{C}$ at $288.12 \mathrm{eV}, \mathrm{C}=\mathrm{O}$ at $289.57 \mathrm{eV}$, respectively and no graphite spectra presented [25]. At the same time, individual peak did not show at $286.74 \mathrm{eV}$ and at $288.12 \mathrm{eV}$,
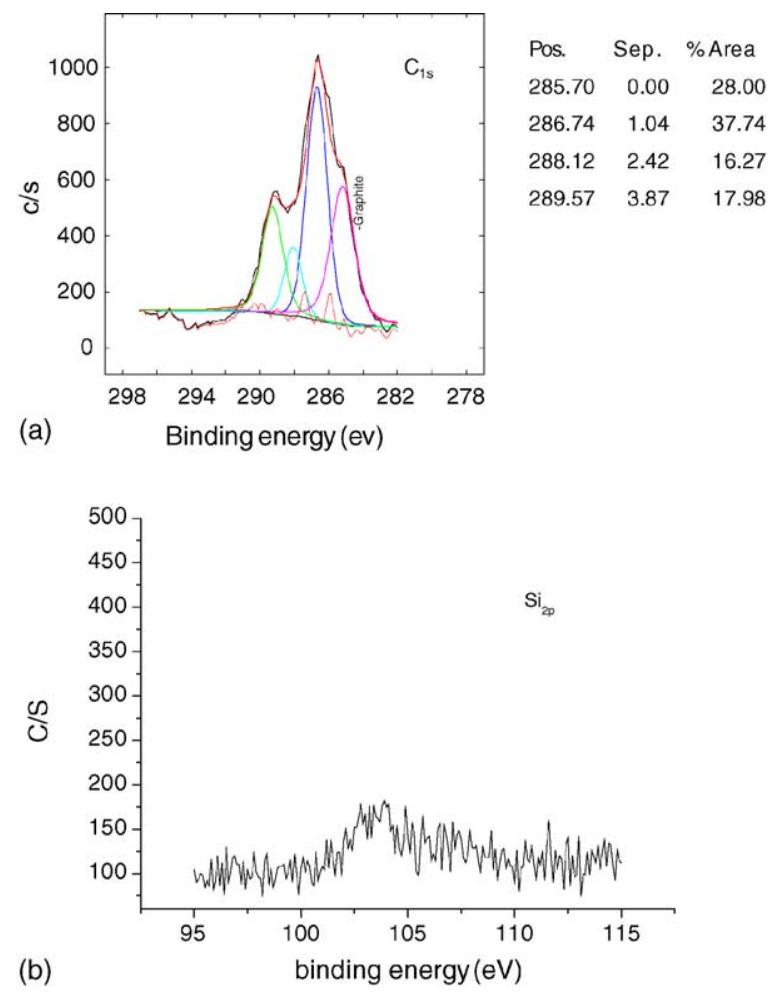

Fig. 5. XPS spectra of STA021: (a) $\mathrm{C}_{1 \mathrm{~s}}$ sprctrium, (b) $\mathrm{Si}_{2 \mathrm{p}}$ spectrium. however presented at $289.57 \mathrm{eV}$, showing chemical environment of $\mathrm{C}-\mathrm{OH}$ and $\mathrm{C}-\mathrm{O}-\mathrm{C}$ was not unitary, but $\mathrm{C}=\mathrm{O}$ was unitary. Analysis of $\mathrm{O}_{1 \mathrm{~s}}$ illustrated that it was $\mathrm{C}-\mathrm{O}-\mathrm{C}$ (aromatic) at $532.92 \mathrm{eV}$ and $\mathrm{C}-\mathrm{OH}$ (aromatic) at $533.50 \mathrm{eV}$. We could summarize from the above analysis that surface layer of composite sorbents was CA layer because graphite spectra representing long chain alkanes of triolein did not appear in $5 \mathrm{~nm}$ surface thickness and XPS extended length of triolein was $29 \AA$ [26]. Spectrum analysis of $\mathrm{Si}_{2 p}$ indicated that silica as a constituent did not appear on the surface of composite sorbent. It was evident that CA or CA/triolein blends had been perfectly deposited on the surface of silica gel.

\subsection{Leakage of the bound chemicals in the composite sorbents}

The cementation degree of CA with silica gel can affect the stability of composite adsorbents. Weak anchor force can make membrane peeled from the silica gel, which nude eyes can see it. However, CA membrane is easily hydrolyzed and encroached by biology in spite of its extensive sources and low prices. Hydrolysis and encroachment of CA will lead to the leakage of triolein into water, accordingly the adsorbed dieldrin releases into water. The secondary pollution will take place. Stability of the composite adsorbents was evaluated by observation with nude eyes and by measuring the triolein in water with photoluminescence method. Linear range of standard curve for triolein was between $22.1365 \mathrm{mg} / \mathrm{l}$ and $132.819 \mathrm{mg} / \mathrm{l}$. Photoluminescence method ensured that triolein could be detected in the case of $0.26 \%$ triolein leakage. Experimental results showed that, to three adsorbents, the phenomenon of being peeled off was not observed and 5\% leakage of triolein was detected in STA021. Thus, it could be seen that triolein content in composite adsorbents should not be too high. Generally, to pure CA membrane spongy holes formed when CA experienced phase transition, while the surface of CA membrane is a compact layer. When triolein was added into the CA syrup, triolein was filled into the holes that formed in the process of phase transition. Low triolein content did not affect the structure of CA membrane, but triolein entered into the compact layer of CA with increased triolein. Accordingly, structure of CA membrane loosened and hydrolysis easily took place. So the experiments about dieldrin adsorption from water were carried out with STA014.

\subsection{Uptake performances of composite sorbents}

Fig. 6 showed changes in the uptake values (dimensionless $C_{t} / C_{0}$ ) of the different sorbents (STA000, STA014, STA021) with time. $C_{t}$ is the dieldrin concentration in water in time $t$. $C_{0}$ is the original concentration. Previous researches have shown that sorption of organic compounds occur in two stages: fast stage, on the time of scale of hours to days, and slow stage, over months to years [27]. The data in Fig. 6 suggested that the removal rate was rapid within $4 \mathrm{~h}$, which was regarded as fast sorption stage. After $24 \mathrm{~h}$ sorption, there was 

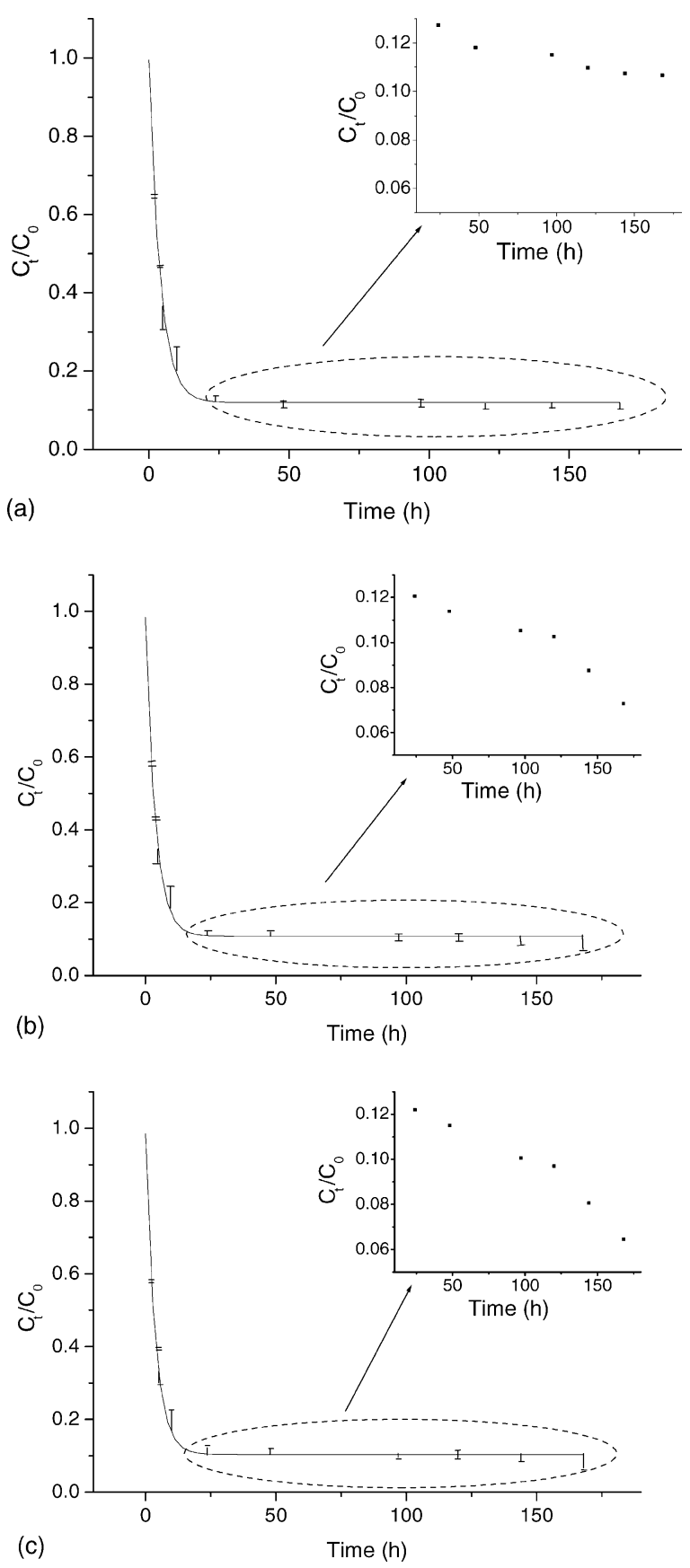

Fig. 6. Effect of different triolein loadings on dieldrin removal from water: $V=0.4 \mathrm{l} ; T=25^{\circ} \mathrm{C} ; N=160 \mathrm{rpm}$; (sorbent) $=10 \mathrm{~g}, C_{0}=200 \mu \mathrm{g} / \mathrm{l}$, (a) STA000, (b) STA014, (c) STA021.

a slow increase in the rate of removal as the experiment proceeded, which was regarded as slow sorption stage. Sorption rate constant $k_{\mathrm{f}}$ and $k_{\mathrm{s}}$ (Table 2) at fast sorption stage and slow sorption stage were obtained by fitting experimental

Table 2

Rate of adsorption at different sorptive stages on different sorbents

\begin{tabular}{lccc}
\hline Adsorbent & STA000 & STA014 & STA021 \\
\hline$k_{\mathrm{f}}\left(\mathrm{h}^{-1}\right)$ & 26.669 & 26.497 & 26.71 \\
$k_{\mathrm{S}}\left(\mathrm{h}^{-1}\right)$ & 0.0273 & 0.06 & 0.0749 \\
\hline
\end{tabular}

data using the Eq. (1) and the Eq. (2).

$$
\begin{aligned}
& \frac{C_{t}}{C_{0}}=-k_{\mathrm{f}} t \\
& \frac{C_{t}}{C_{0}}=-k_{\mathrm{s}} t
\end{aligned}
$$

From Table 2, it was shown that fast sorption rate had nothing to do with increased triolein, however, the result at slow sorption stage was different from that at fast sorption stage. The slow sorption rate apparently increased due to the increase of the triolein content (inset in Fig. 6a-c). Sorption rate increased from 0.0273 to 0.0749 when triolein/CA ratio increased from 0 to 0.21 . Thus, it could be seen that slow sorption stage was the major stage at which triolein could affect the dieldrin removal. The needed time of adsorption equilibrium prolonged with increased triolein contents.

The results of sorption experiments could be explain from the structure of sorbents. These sorbents had the same outer surface of CA and triolein as major adsorptive material was embedded into CA. Internal silica gel hardly has adsorptive capability (experimental result no shown). Dieldrin was firstly adsorbed onto surface of the adsorbents, and then transferred into channels of CA, finally was adsorbed into the triolein. At the beginning of adsorption, drive force is the dieldrin concentration difference between the surface of the adsorbents and the bulk solution. Due to the same external surface of three different sorbents and the surrounding fluid concentration, there was the same concentration difference across the boundary layer around each particle, that was, the same driving force. So three different sorbents had the same $k_{\mathrm{f}}$. With prolonged time, dieldrin that was adsorbed onto the surface of adsorbents gradually transferred into the CA channels. Here made a presumption that the adsorption from CA to triolein is fast. Dieldrin in channels fastly transferred into triolein, transfer speed quicken with the increased triolein content, accordingly leave more adsorptive positions in CA.

Subsequently, Uptake experiments of $50 \mathrm{ng} / \mathrm{l}$ dieldrin were carried out with composite sorbent STA014 (Fig. 7) in order to test the removal capability from actual water bodies. $U$ in Fig. 7, the removal fraction, calculated according to Eq. (3).

$U=\frac{1-C_{t}}{C_{0}}$

$C_{t}$ is the dieldrin concentration in water in time $t$, and $C_{0}$ is the original concentration. Plot in Fig. 7 showed the same results as Fig. 6. Dieldrin removal rate attained to $80 \%$, which identified good removing efficiency. But initial fast sorption stage extended to $24 \mathrm{~h}$. It was evident that drive force of adsorption was positively related to the dieldrin concentration in bulk solution. Decreased diedrin concentration in bulk solution slowed down the adsorptive rate. However, because the adsorptive capacity was positively related to triolein content, the adsorptive capacity hardly changes. 


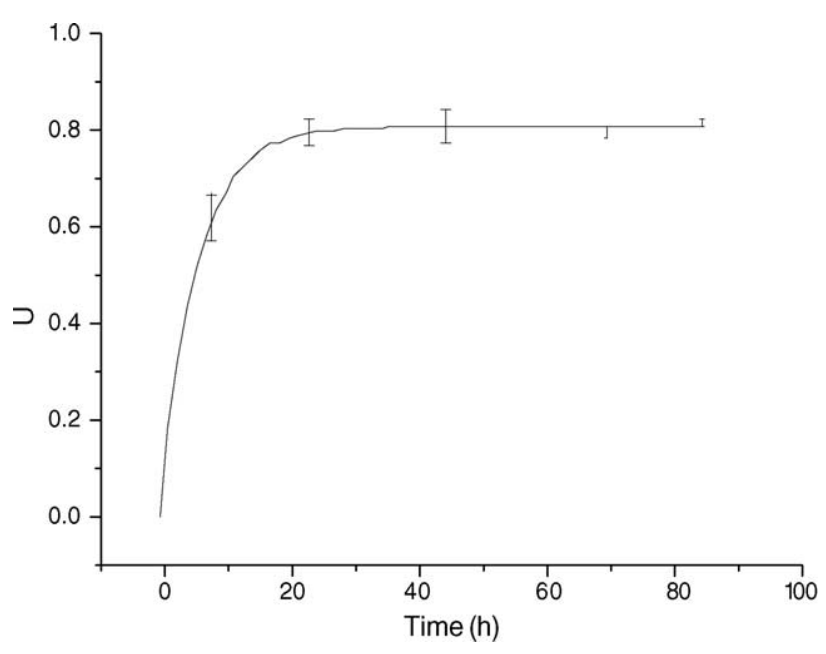

Fig. 7. Time dependence on dieldrin removal from water for STA014: $V=0.4$ 1; $T=25^{\circ} \mathrm{C} ; N=160 \mathrm{rpm}$; (sorbent) $=10 \mathrm{~g}, C_{0}=50 \mathrm{ng} / \mathrm{l}$.

\section{Conclusions}

SEM of the triolein/CA blends suggested that triolein was effectively embedded into $\mathrm{CA}$ and a compact surface layer containing no triolein formed, which ensured that triolein hardly leaked out from CA membrane. Immiscibility of triolein and CA identified by ATR-FTIR ensured that chemical structure of triolein hardly changed, accordingly the accumulation ability.

XPS analysis of silica gel before and after modification suggested that sonication for $30 \mathrm{~min}$ is needed to complete the modification in order to strengthen the cementation between silica gel and CA.

Comparing the sorptive and desorptive curves of composite adsorbents to that of silica gel, the structure of composite adsorbents is largely different from that of silica gel after silica gel was coated with triolein/CA membrane. XPS of composite adsorbent suggested that silica as a compound disappeared from the surface of composite material, silica gel has been completely coated by triolein/CA hybrid membrane and triolein has perfectly been embedded in CA membrane. Measurement of the leakage of the bound chemicals from composite adsorbents showed that appropriate triolein content did not lead to hydrolysis and encroachment of CA.

Novel composite adsorbents embedded with triolein can effectively help to uptake dieldrin from aqueous solution. Uptake values for dieldrin increase with the increased triolein loading in the membrane.

About $80 \%$ dieldrin can be removed even to nanogram level concentration of dieldrin. However, fast sorption rate of dieldrin is relatively low. Subsequent job must be done to shorten fast sorption process.

\section{Acknowledgements}

The authors appreciate the generous financial support of this work by National High-tech Research and Development Plan (No. 2002AA649080) and important foundation from National Natural Science Foundation of China (No. 20337020).

\section{References}

[1] B. Jansson, In International Symposium on Environmental Endocrine Disrupters, 2001. Available from http://www.env.gojp/chemi/ end/2001 report/pdf-e/jansson-e.pdf.

[2] A. Evenset, G.N. Christensen, T. Skofvold, Sci. Total Environ. 318 (2004) 125-141.

[3] P.K.K. Louie, D.W. Sin, Chemosphere 52 (2003) 1397-1403.

[4] Z.L. Zhang, H.S. Hong, J.L. Zhou, J. Huang, G. Yu, Chemosphere 52 (2003) 1423-1430.

[5] Detlef Bahnemann, Solar Energy 77 (2004) 445-459.

[6] Urs von Gunten, Water Res. 37 (2003) 1443-1467.

[7] Li Xiao-yan, Chu Hiu Ping, Water Res. 37 (2003) 4781-4791.

[8] X. Fan, D.J. Parker, M.D. Smith, Water Res. 37 (2003) 4929-4937.

[9] Gurusamy Annadurai, Ruey-Shin Juang, Duu-Jong Lee, Adv. Environ. Res. 6 (2002) 191-198.

[10] Z.S. Wang, W.J. Liu, Drinking Water Treatments for Micropollutant Water Resources, Construction Industry Press, Beijing, 2001, p. 62.

[11] C.M. John, R.E. Kathy, J.N. Huckins, Environ. Sci. Technol. 32 (1998) 1847-1852.

[12] W.G. Robert, J.N. Huckins, J.D. Petty, Environ. Sci. Technol. 31 (1997) 178-187.

[13] J.D. Petty, J.N. Huckins, C.E. Orazio, Environ. Sci. Technol. 29 (1995) 2561-2566.

[14] C.S. Hofelt, D. Shea, Environ. Sci. Technol. 31 (1997) 154-159.

[15] Kees Booij, B.L. Drooge, Chemosphere 44 (2001) 91-98.

[16] Y.B. Lu, Z.J. Wang, Water Res. 37 (2003) 2419-2425.

[17] L. Huang, H. Xiao, Y. Ni, Colloids Surf. A Physicochem. Eng. Aspects 247 (2004) 129-136.

[18] Anyun Zhang, Yuezhou Wei, Mikio Kumagai, React. Funct. Polym. 61 (2004) 191-202.

[19] Sou-Yee Mak, Dong-Hwang Chen, Dyes Pigments 61 (2004) 93-98.

[20] C.T. Chiou, Environ. Sci. Technol. 19 (1985) 57-62.

[21] C.T. Chiou, V.H. Freed, Environ. Sci. Technol. 11 (1977) 475-478.

[22] A.M. Lazarin, C.A. Borgo, J. Membr. Sci. 221 (2003) 175-184.

[23] B.D. Reid, R.T. Alberto, Chem. Mater. 13 (2001) 2366-2373.

[24] F. Ruo, L. Huamao, Phonochemistry and its Application, Science and Technology Press, Hefei, 1992.

[25] D. Briggs, Surface Analysis of Polymers by XPS and Static SIMS Engineering, Chemical Industry Press, Beijing, 2001, p. 70.

[26] M. Donald, Small, Handbook of Lipid Research 4, Plenum Press, New York, 1986, p. 370.

[27] Tiziana Missana, César Maffiotte, Miguel García-Gutiérrez, J. Colloid Interface Sci. 261 (2003) 154-160. 